\title{
Psychotherapy research: linking efficacy to policy and practice
}

\author{
GLENYS PARRY AND ANTHONY D. ROTH
}

\section{THE POLICY CONTEXT}

The cost and effectiveness of psychological therapies in mental health services are both important and topical. Psychotherapies are commonly practised, there is growing recognition of the psychological aspects of health, and increasing public awareness of the role of «talking therapies». Internationally, the costs of health care have increased and will continue to do so, as available health technologies proliferate and public expectations of better health services rise. Those responsible for third party payment in health care, whether payment by Government revenue, health insurance or employers, are concerned with cost containment. There has been a parallel concern to base health policy and management decisions on evidence of clinical effectiveness and to promote better standards of health care interventions (Muir Gray, 1997). "Managed care» initiatives, particularly in the USA, have attempted to address both concerns through the greater use of clinical guidelines and reimbursement decisions to change clinician behaviour (Schwartz \& Brennan, 1996). These initiatives are controversial; concern has been expressed by some clinicians that they damage the relationship between clinician and patient and can paradoxically lower standards of care, whereas others have accommodated to them and have changed their clinical practices in response (Berman \& Austad, 1997). The debate suggests that such moves leave many clinicians suspicious of demands for evidence based

Indirizzo per la corrispondenza: Professor G. Parry, University of Sheffield, School of Health \& Related Research, 30 Regent Street, Sheffield S1 4DA (UK).

Fax + 44-114-272.4095

E-mail: G.D.Parry@sheffield.ac.uk practice, and generates adversarial processes between funders, clinicians and researchers.

In 1996 the English Department of Health published a new policy on good practice in psychotherapy services (Department of Health, 1996). As part of this process, the Department commissioned a review of research evidence on psychotherapy efficacy and effectiveness (Roth \& Fonagy, 1996). In this Editorial, we draw on our work for the policy and research reviews respectively.

The Department of Health policy review recommends caution in basing funding decisions solely on the existing base of research evidence in this field, arguing that there is a notable absence of research on some widely practised treatments; the weaknesses of psychotherapy research in relation to external validity are severe; research compares treatments within diagnostic groups, whereas other factors will govern the clinical choice of therapy, such as the capacity to form a therapeutic alliance, or the level of social support available to the individual; outcome measurement in this research continues to be problematic, e.g. in relation to levels of functioning or quality of life, and although well designed group comparisons can yield results which are generally true, the specific applicability of these findings to the individual case remains a matter for clinical judgement.

In our view, either uncritical rejection or acceptance of research findings is inappropriate, partly because both stances ignore the fact that researchers, clinicians and funders have different perspectives on research and different needs in relation to it. In this article we broadly outline the state of knowledge about the efficacy of psychological therapies and present a model for evidence based psychotherapy practice which we believe reconciles these different viewpoints. 


\section{RESEARCH ON THE EFFICACY OF PSYCHOTHERAPY}

Roth \& Fonagy (1996) collated studies of the efficacy of therapies in relation to mental health conditions commonly seen in clinical settings. Overall they found good evidence for the efficacy of psychological therapies. Evidence for the differential efficacy of therapies was not strong, though this conclusion is tempered by the fact that some therapies are less well researched than others, and the quality of the research evidence often varies across orientations. Thus, while there are many studies of behavioural and cognitive behavioural therapies, those examining the efficacy of psychodynamic approaches are often limited, partly by the paucity of trials, and also because a number of these studies were conducted by proponents of alternative therapies, with the result that the psychodynamic therapy on offer is offered by inexperienced therapists.

Despite a general conclusion that there is only modest evidence of a differential effect between therapies, there are instances where more certain therapeutic recommendations can be made. Thus while the evidence that interpersonal therapy, cognitive behavioural therapy and brief psychodynamic therapies can all be beneficial in the treatment of depression, there is reasonably strong evidence for the superior benefits of cognitive behavioural therapies in generalised anxiety disorder, for the use of interpersonal therapy in eating disorders, and of panic control therapies in relation to panic disorder without agoraphobia.

Outcomes expected from a psychological intervention will vary in relation to the presenting problem, and care should be taken neither to over or understate the likely benefits of treatment. Often outcomes will be moderate, though clinically significant - for example, data from a number of trials (Elkin, 1994; Hollon et al., 1992; Shapiro et al., 1994) suggest that after brief intervention, approximately $50 \%$ of patents presenting with acute depression will reach a recovery criterion. In other presentations a higher rate of recovery may be likely - for example Clark et al. (1994) treating patients with panic disorder without agoraphobia, had a recovery rate of $90 \%$ at one-year follow-up. For other conditions rates of recovery may be low, as appears to be the case in some of the sexual dysfunctions.

Not all patients will benefit from short-term treatment - indeed the natural history of some disorders suggests that they follow a cyclic path of remission and relapse which therapy may ameliorate, rather than abolish. Thus, while brief treatments are often effective, they may not be sufficient in longer-term management. For many patients a maintenance model may be useful, within which acute treatment is followed by planned but intermittent contact. In the University of Pittsburgh study (Frank et al., 1990) this pattern of care significantly delayed or even prevented further episodes in depressed individuals with a prior history of remission and relapse.

Though sometimes seen as incompatible or even alternative forms of treatment, there is good evidence that medication and psychotherapy can be seen as adjunctive and complementary, though their relative roles may differ between conditions. Thus in depression there is good evidence for their complementary use, while there appears to be little additional benefit to medication in panic disorder with agoraphobia. For other conditions, such as schizophrenia, clinicians would be ill-advised to use psychological interventions in the absence of medication, despite the known efficacy of family treatments for this group.

Despite the apparent certainty of the above, there insufficient evidence to allow the prescription of different therapies on the basis of diagnosis. "Brand names» are only partially predictive, if at all, and as discussed below, the therapeutic alliance is an important, though complex, factor in outcome. Therapists differ in their skilfulness; in some trials outcome variance attributable to therapists is greater than that contributed by therapeutic method. Manualisation of therapies, while assuring some degree of consistency in treatment delivery, remains controversial (Persons, 1991) and of doubtful utility beyond research trials.

\section{RESEARCH FOR PRACTICE; A MODEL OF RESEARCH, EDUCATION AND AUDIT}

Figure 1 gives a model of evidence-based practice in psychotherapy services (Department of Health, 1996; Roth \& Fonagy, 1996). It shows the appropriate relationships between research, standard practice, developments in practice, service evaluation, theoretical developments, clinical audit and professional training and education. All these activities should work systematically together to improve the quality and effectiveness of psychotherapy services to patients. 


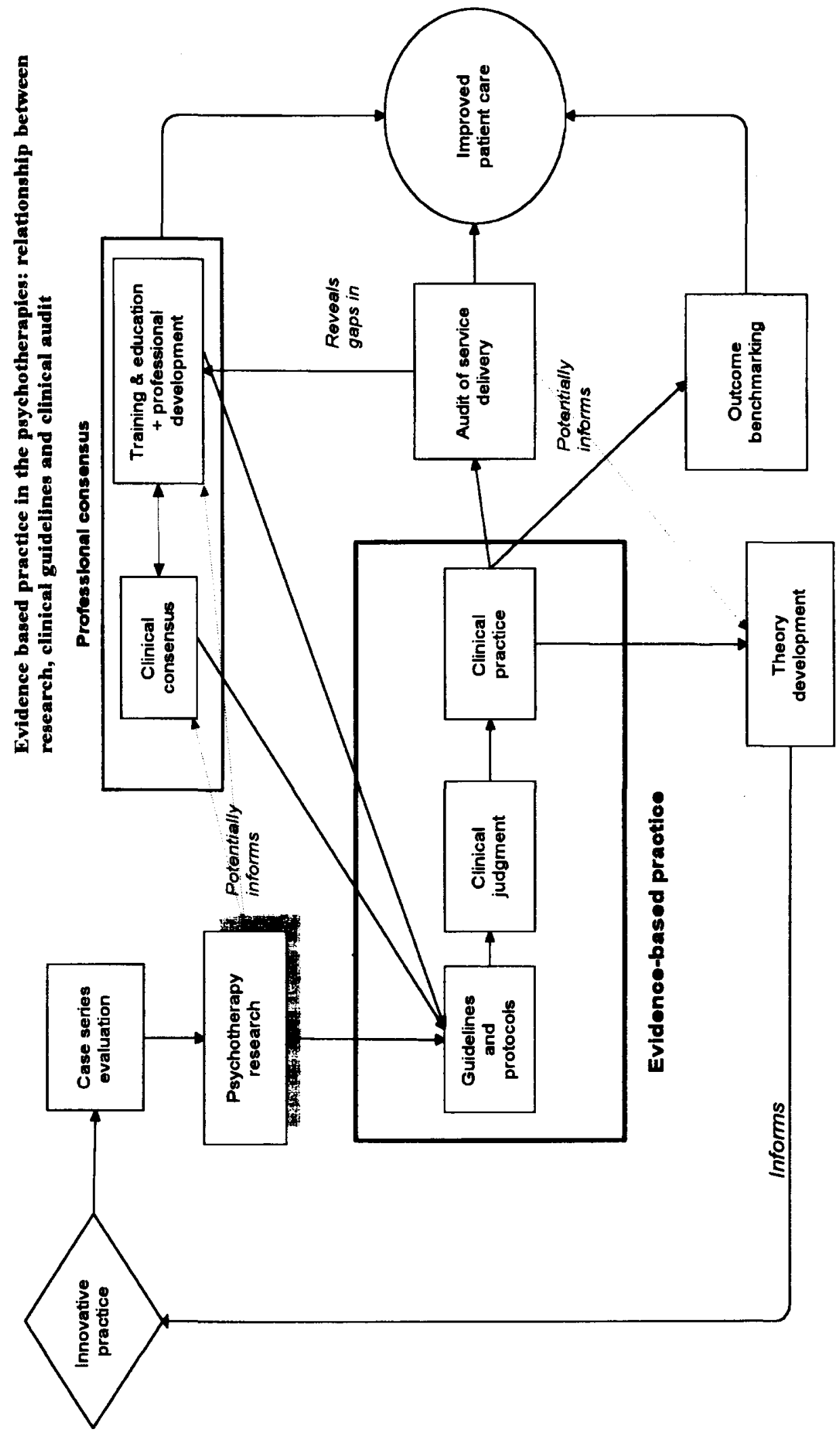

Epidemiologia e Psichiatria Sociale, 6, 2, 1997 
The model has a fundamental tenet that standard practice in delivering psychotherapies could be far more clearly specified through clinical guidelines than is currently the case. Psychottherapists are wary of the concept of clinical protocols and guidelines, seeing them as a threat to clinical freedom, but clinical guidelines can be used to benchmark best practice and as an aid to clinical judgement. They should be informed by service evaluation evidence, formal research findings and clinical consensus. Research evidence is incorporated into standard clinical practice through these protocols, but it is the prerogative of the clinician to make a judgement in the individual case, on the basis of assessment and case formulation, to do something different. However, the guideline acts as an aide-memoire to the therapist in formalising normal expected practice, so that deviations from this practice are made thoughtfully and are justified by clinical evidence.

Though an emphasis on standardised practice might be seen as a threat to innovation, in practice this is unlikely. There will always be many situations where research evidence - or even clinical consensus - cannot indicate the best or most effective approach. Solutions to clinical impasses can be suggested by the failure of systematically applied and monitored standard practice, and hence such practice can be seen as a springboard to new developments, which themselves become part of the cycle of research activity. Initially, novel forms of service delivery or experimental clinical techniques are likely to be tested through case studies or replicated $n=1$ evaluation of case series. If the technique or approach appears promising, randomised controlled trials should be undertaken to establish whether or not the treatment is efficacious, and larger scale field trials carried out to evaluate its clinical effectiveness, as delivered in everyday practice.

Standard practice which can be described in clinical guidelines also has the great advantage of focusing and clarifying the audit effort, since the guideline states "the right thing to do" whereas the audit asks "has the right thing been done?» and "has it been done right?» By targeting audit onto those processes derived from evidence-based protocols, a clear link can be established between research and audit. In turn, this should reveal gaps in knowledge, leading to developments in theory and new hypotheses for formal psychotherapy research.

As clinical and service audit examines the reasons for failures to deliver to a set standard of practice, it can reveal gaps in the skills and competence of psy- chotherapists which are preventing them delivering a fully effective service. This highlights quality of professional training, new education and curriculum needs, and integrated attention to questions of professional skills updating. It is likely that it is only by following such a process of service delivery, that both pre and post-qualification training in the psychotherapies will become underpinned by links among theory, research and practice.

\section{BEYOND TREATMENT TRIALS; RESEARCH TOPICS FOR IMPROVED CLINICAL EFFECTIVENESS}

The type of psychotherapy intervention alone is not the strongest predictor of outcome. Current research directs attention to characteristics of patients and therapists, specifically, the interaction between client and treatment characteristics, known as Aptitude Treatment Interaction (ATI) research (Shoham-Salomon, 1991), and to the interplay between therapist and patient, as represented by the therapeutic alliance. One therapist characteristic which has become a focus for operational definition and research investigation is competence. The latter two research directions are reviewed below. Together these topics provide an opportunity to develop and research treatment-of-choice guidelines, following from treatment recommendations for single conditions, for example depression, where clinical practice guidelines already exist (American Psychiatric Association, 1993; Depression Guideline Panel, 1993). Although such guidelines have attracted criticism on a number of counts, including the charge that their prescriptions go beyond the evidence (Persons et al., 1996), a clinical practice guideline to aid treatment-of-choice decisions would integrate evidence from comparative outcome trials between therapy types for specific diagnoses, with evidence relating to the therapeutic alliance, treatment-aptitude interactions and therapeutic competence. This holds out the possibility, long forseen but not yet realised, of achieving the best fit between patient, therapist and treatment on the basis of evidence of efficacy across theoretical orientations, using multiple diagnostic and non-diagnostic indications (Norcross, 1995; Beutler \& Clarkin, 1990; Frances et al., 1984). The impact of practice guidelines on the clinical effectiveness of services requires robust routine measurement 
of patient progress and outcomes in therapy as delivered to whole clinical populations, as well as the selected samples within randomised clinical trials (Sperry et al., 1996).

\section{THERAPEUTIC ALLIANCE AND THERAPIST COMPETENCE}

In a research context, the alliance is a pantheoretical concept comprising three related elements; the degree to which the patient and therapist are bonded (for example, through an experience and expression of mutual respect), the extent to which they agree on the goals of therapy, and the degree to which they agree on the relevance and the appropriateness of the tasks being used to reach these goals.

Research instruments to measure the quality of the alliance usually rate audio-recordings of therapy using scales that distinguish the contribution to the alliance of the patient, therapist or the two in interaction (Horvath \& Greenberg, 1989). Alternatively, therapists and patients are asked to rate their sense of the alliance directly. Meta-analytic review of an increasingly substantial body of research (Horvath \& Symonds, 1991) demonstrates the significant contribution of the alliance to outcome, yielding an effect-size of $0.2-0.3$ for the early and late alliance respectively. Given the difficulty of relating process variables to outcome, and the authors' bias towards caution, this apparently modest effect-size is impressive.

The significance of the alliance is further emphasised by recently published data from two trials (NIMH trial, Elkin, 1994; University of Pittsburgh trials, Hollon et al., 1992), showing that the alliance was positively associated with outcome, an effect seen across differing therapeutic methods, including pharmacotherapy (Krupnick, et al., 1996; Castonguay et al., 1996).

There has been a developing research focus on threats to the alliance, both as a marker for mutative events within therapy, and as an indicator of therapist skilfulness. A number of workers have suggested that patient challenges to the alliance will reflect aspects of their underlying pathology, and that these junctures represent an opportunity for therapeutic change - and also for the temporary or permanent loss of the patient to therapy (Safran \& Muran, 1996). Work in progress attempts to model the management of these events, using task analysis and statistical mapping (Safran \& Muran, 1996; Sexton et al., 1996; Kivlighna \& Shaughnessy, 1995). Inevitably such work directs attention to the skilfulness with which therapists manage the alliance.

Aveline et al. (1995) see measurement of therapist competence as a research priority. Attention to the skilfulness of therapists is vital in translating research findings on efficacy to clinically effective services and there is a need for better measures of therapist competence, in psychotherapy research, practice and training. Psychotherapy researchers are now well aware of the scientific requirement to check that therapists adhere to a specific treatment approach, as defined in a manual, but they must also ensure that the therapy was delivered competently and therefore had a fair trial. Clinicians need audit tools to help them improve the quality of services and those involved in training need to evaluate the performance of those qualifying and assess the overall effectiveness of the training.

Although there has generally been neglect of the contribution of therapist skill in treatment outcome research (Moncher \& Prinz, 1991), there is some evidence that skilfulness predicts good outcome (CritsChristoph et al., 1988). However, the relationship between training and skilfulness is weaker than commonly assumed (Garb, 1989; Peterson, 1995). Although supervision enhances therapist confidence, there is little evidence that supervision and practice alone produce competence in psychotherapy (Beutler, 1995; Guest \& Beutler, 1988). There is also only modest support for the benefits of experience (Roth \& Fonagy, 1996). In part this may be a methodological problem - differential attrition between the patients of novice and experienced therapists may lead to the former losing, and the latter treating, more difficult patients, hence yielding apparently poorer outcomes for the more experienced therapists. Although, in the absence of longitudinal studies, the impact of experience remains uncertain, it is clear that experience is not equivalent to competence.

Therapist competence encompasses both non-specific and technique-specific skills (Rounsaville et al., 1987). Non-specific skills can be captured by measurement of the rate of therapist errors (Suh et al., 1986), but measurement of technique-specific skills must specify criteria of competence which go beyond treatment model adherence (Waltz et al., 1993). Although there is a high correlation between adherence and competence, it is quite possible to adhere to the requirements of a specific technique to 
the detriment of other therapeutic factors; indeed, there is evidence of the negative impact of practising technique in the absence of the alliance, an effect demonstrated in relation both to cognitive and psychodynamic therapy. Thus Castonguay et al. (1996) found that increased use of cognitive technique was associated with poorer outcomes, though this effect was abolished if the alliance was controlled for. This finding echoes that of Henry et al. (1993), who found that in increasing their technical competence, trainee psychodynamic therapists neglected to attend to the alliance, to the detriment of outcomes.

Process-based definitions of competence emphasise performance and behaviour, often rating aspects of an individual's performance against a model of a skilful performance. Measures of this type have been developed for cognitive therapy (Dobson et al., 1985) and interpersonal therapy (Klerman et al., 1984). An alternative process oriented method takes expert case formulations and experts also rate the appropriateness of psychoanalytic interventions on the basis of these (Silberschatz et al., 1986). Waltz et al. (1993) criticise the assumption that therapists will demonstrate a uniformity of competence across clients and problems. They strongly recommend researchers to understand better the contextual features which influence skilfulness.

The difficulties are not solved by defining competence in terms of outcomes; that is, defining as competent any therapist who consistently achieves good outcomes, or who performs normatively in terms of therapeutic outcomes for a particular client group. The relationship between therapist behaviour and outcome is weak, and it can be argued that one would not logically expect to see a linear relationship between the amount of a given active ingredient' in therapy and the therapeutic outcome, because therapists adapt and respond to the needs of the patient (Stiles \& Shapiro, 1994).

Some evidence that competence relates to outcome reflects the success of the therapist-patient dyad in establishing a working relationship. For example, in Franks' studies of long-term maintenance therapy for depression (Frank et al., 1991), patients receiving therapy rated as competently delivered, had a median survival time to relapse of two years, contrasted to a median survival time of 4 months in those receiving poor-quality therapy. However therapists in this study were able to deliver competent therapy with only some of their patients; other of their patients received poor quality therapy. This sort of result inevitably draws attention back to the therapeu- tic alliance. Although it is clear that patients vary considerably in their capacity to form alliances (Piper et al., 1991; Mallinckrodt et al., 1995), one index of the competent therapist might be their capacity to draw the more disturbed and distrusting patient into a working relationship.

Research on the therapeutic alliance and therapist skill has important implications for how psychotherapists are trained and accredited. Training which uses targeted goals, specific feedback and guided practice in order to enable therapists to reach a stated criterion of competence is likely to be most effective (Luborsky, 1990). Beutler et al. (1994) call for more rigorous and specific training methods than current supervision models provide, suggesting that competency based training using therapy manuals may provide such an alternative. This method has been adopted to some extent in cognitive and behavioural therapy training (Bootzin \& Ruggill, 1988; Dobson \& Shaw, 1988; Dobson et al., 1985; Shaw \& Dobson, 1988) and although there are examples in psychodynamic therapy (Moss et al., 1991; Strupp et al., 1988), measures of specific psychodynamic competencies are not yet widely available.

\section{REFERENCES}

American Psychiatric Association (1993). Practice guideline for major depressive disorder in adults. American Journal of Psychiatry 150, 1-26.

Aveline M., Shapiro D.A., Parry G.\& Freeman C.P.L. (1995). Building research foundations for psychotherapy practice. In Research Foundations for Psychotherapy Practice (ed. M. Aveline and D.A. Shapiro), Chapter 14. Wiley: Chichester.

Berman W.H. \& Austad C.S. (1997). Managed mental health care: current status and future directions. In Psychotherapy in Managed Health Care; The Optimal Use of Time and Resources (ed. C.S. Austad and W.H. Berman), pp. 264-278. American Psychological Association: Washington DC.

Beutler L.E. (1995). The germ theory myth and the myth of outcome heterogeneity. Psychotherapy 32, 489-494.

Beutler L.E. \& Clarkin J.F. (1990). Systematic Treatment Selection. Brunner/Mazel: New York.

Beutler L.E., Machado P.P.P. \& Neufeldt S.A. (1994). Therapist variables. In Handbook of Psychotherapy and Behavior Change, 4th ed. (ed. A.E. Bergin and S.L. Garfield), pp. 229-269. Wiley: New York.

Bootzin R.R. \& Ruggill J.S. (1988). Training issues in behavior therapy. Journal of Consulting and Clinical Psychology 56, 703-709.

Castonguay L.G., Goldfried M.R., Wiser S., Raue P.J. \& Hayes A.M. (1996). Predicting the effect of cognitive therapy for de- 
pression: a study of unique and common factors. Journal of Consulting and Clinical Psychology 64, 497-504.

Clark D.M., Salkovskis P.M., Hackmann A., Middleton H., Anastasiades P. \& Gelder M. (1994). A comparison of cognitive therapy, applied relaxation and imipramine in the treatment of panic disorder. British Journal of Psychiatry 164, 759-769.

Crits-Christoph P., Cooper A. \& Luborsky L. (1988). The accuracy of therapists' interpretations and the outcome of dynamic psychotherapy. Journal of Consulting and Clinical Psychology $56,490-495$.

Department of Health (1996). A Review of Strategic Policy on NHS Psychotherapy Services in England. NHS Executive: London.

Depression Guideline Panel (1993). Clinical Practice Guideline No 5; Depression in Primary Care. 2: Treatment of Major Depression. AHCPR 93-551 Agency for Health Care Policy \& Research: Rockville MD.

Dobson K.S. \& Shaw B.F. (1988). The use of treatment manuals in cognitive therapy: Experience and issues. Journal of Consulting and Clinical Psychology 56, 673-680.

Dobson K.S., Shaw B.F. \& Vallis T.M. (1985). Reliability of a measure of the quality of cognitive therapy. British Journal of Clinical Psychology 24, 295-300.

Elkin I. (1994) The NIMH treatment of depression collaborative research programme: where we began and where we are. Chapter 4. In Handbook of Psychotherapy and Behaviour Change, 4th ed. (ed. A.E. Bergin and S.L. Garfield), pp. 114-139. Wiley: New York.

Frances A., Clarkin J.F. \& Perry S. (1984). Differential Therapeutics in Psychiatry: The Art and Science of Treatment Selection. Brunner/Mazel: New York.

Frank E., Kupfer D.J., Perel J.M., Jarrett D.B., Mallinger A.G., Thase M.E., McEachran A.B. \& Grochocinski C.J. (1990). Three year outcomes for maintenance therapies in recurrent depression. Archives of General Psychiatry 47, 1093-1099.

Frank E., Kupfer D.J., Wagner E.F., McEachran A.B. \& Cornes C. (1991). Efficacy of interpersonal therapy as a maintenance treatment of recurrent depression. Archives of General Psychiatry 48, 1053-1059.

Garb H.N. (1989). Clinical judgment, clinical training and professional experience. Psychological Bulletin 105, 387-396.

Guest P.D. \& Beutler L.E. (1988). The impact of psychotherapy supervision on therapist orientation and values. Journal of Consulting and Clinical Psychology 56, 653-658.

Henry W.P., Strupp H.H., Butler S.S., Schact T.E. \& Binder J.L. (1993). Effects of training in time limited dynamic psychotherapy: changes in therapist behavior. Journal of Consulting and Clinical Psychology 61, 434-440.

Hollon S.D., Du Rubeis R.J., Evans M.D., Weimer M.J., Garvey M.J., Grove W.M. \& Tuason V.B. (1992). Cognitive therapy and pharmacotherapy for depression: Singly or in combination. Archives of General Psychiatry 49, 774-781.

Horvath A.O. \& Greenberg L.S. (1989). Development and validation of the working alliance inventory. Journal of Counselling Psychology 36, 223-233.

Horvath A.O.\& Symonds B.D. (1991). Relation between working alliance and outcome in psychotherapy: A meta-analysis. Journal of Consulting and Clinical Psychology 38, 139-149.

Kivlighna D.M. \& Shaughnessy P. (1995). Analysis of the develop- ment of the working alliance using hierarchical linear modelling. Journal of Counselling Psychology 42, 338-349.

Klerman G.L., Weissman M.M., Rounsaville B.J. et al. (1984). Interpersonal Psychotherapy of Depression. Basic Books: New York.

Krupnick J.L., Sotski S.M., Simmens S., Moyer J., Elkin I., Watkins J. \& Pilkonis P.A. (1996). The role of the therapeutic alliance in psychotherapy and pharmacotherapy outcome: Findings in the NIMH treatment of depression collaborative research programme. Journal of Consulting and Clinical Psycho$\log y$ 64, 532-539.

Luborsky L. (1990). Theory and technique in dynamic psychotherapy. Curative factors and training therapists to maximize them. Psychotherapy and Psychosomatics 53, 50-57.

Mallinckrodt B., Gantt D.L. \& Coble H.M. (1995). Attachment patterns in the psychotherapy relationship: Development of the client attachment to therapist scale. Journal of Counselling Psychology 42, 307-317.

Moncher F.J. \& Prinz R.J. (1991). Treatment fidelity in outcome studies. Clinical Psychology Review 11, 247-266.

Moss S., Margison F. \& Godbert K. (1991). The maintenance of psychotherapy skill acquisition: a two-year follow-up. British Journal of Medical Psychology 64, 233-236.

Muir Gray J.A. (1997). Evidence-Based Healthcare. How to Make Health Policy and Management Decisions. Churchill Livingstone: New York.

Norcross J.C. (1995). Dispelling the dodo bird verdict and the exclusivity myth in psychotherapy. Psychotherapy 32, 500-504.

Persons J.B. (1991). Psychotherapy outcome studies do not accurately represent current models of psychotherapy: A proposed remedy. American Psychologist 46, 99-106.

Persons J.B., Thase M.E. \& Crits-Christoph P. (1996). The role of psychotherapy in the treatment of depression. Archives of $\mathrm{Ge}$ neral Psychiatry 53, 283-290.

Peterson D.R. (1995). The reflective educator. American Psychologist 50, 975-983.

Piper W.E., Azim H.F.A., Joyce A.S., McCallum M., Nixon G.W.H. \& Segal P.S. (1991). Quality of object relations versus interpersonal functioning as predictors of therapeutic alliance and psychotherapy outcome. Journal of Nervous \& Mental Disease 179, 432-438.

Roth A.D. \& Fonagy P. (1996). What Works for Whom? A Critical Review of Psychotherapy Research. Guilford Press: New York.

Rounsaville B.J., Chevron E.S., Prusoff B.A., Elkin I., Imber S. \& Watkins J. (1987). The relation between specific and general dimensions of the psychotherapy process in interpersonal psychotherapy of depression. Journal of Consulting and Clinical Psychology 55, 379-384.

Safran J.D. \& Muran J.C. (1996). The resolution of ruptures in the therapeutic alliance. Journal of Consulting and Clinical Psycho$\log y$ 64, 447-458.

Schwartz K. \& Brennan T.A. (1996). Integrated health care, capitated payment, and quality: the role of regulation. Annals of Internal Medicine 124, 442-448.

Sexton H.C., Hembre K. \& Kvarne G. (1996). The interaction of the alliance and therapy microprocess: A sequential analysis. Journal of Consulting and Clinical Psychology 64, 471-480.

Shapiro D.A., Barkham M., Rees A., Hardy G.E., Reynolds S. \& Startup M. (1994). Effects of treatment duration and severity of depression on the effectiveness of cognitive/behavioural

Epidemiologia e Psichiatria Sociale, 6, 2, 1997 
and psychodynamic/interpersonal psychotherapy. Journal of Consulting and Clinical Psychology 62, 522-534.

Shaw B.F. \& Dobson K.S. (1988). Competency judgements in the training and evaluation of psychotherapists. Journal of Consulting and Clinical Psychology 56, 666-672.

Shoham-Salomon V. (1991). Introduction to special section on client-therapy interaction research. Journal of Consulting and Clinical Psychology 59, 203-204.

Silberschatz G., Fretter P.B.\& Curtis J.T. (1986). How do interpretations influence the process of psychotherapy? Journal of Consulting and Clinical Psychology 54, 646-652.

Sperry L., Brill P.L., Howard K.I. et al. (1996). Treatment Outcomes in Psychotherapy and Psychiatric Interventions. Brunner/ Mazel: New York.

Stiles W.B. \& Shapiro D.A. (1994). Disabuse of the drug meta- phor: psychotherapy process outcome correlations. Journal of Consulting and Clinical Psychology 62, 942-948.

Strupp H.H., Butler S.F. \& Rosser C. (1988). Training in psychodynamic therapy. Journal of Consulting and Clinical Psychology 56, 689-695.

Suh C.S., O'Malley S.S. \& Strupp H.H. (1986). The Vanderbilt process measures: The Psychotherapy Process Scale (VPPS) and the Negative Indicators Scale (VNIS). In The Psychotherapeutic Process: A Research Handbook (ed. L.S. Greenberg and W.M. Pinsof), pp. 285-323. Guilford Press: New York.

Waltz J., Addis M.E., Koerner K. \& Jacobson N.S. (1993). Testing the integrity of a psychotherapy protocol: Assessment of adherence and competence. Journal of Consulting and Clinical Psychology 61, 620-630. 